\title{
Nonbacterial thrombotic endocarditis associated with chronic hypoxia and secondary polycythemia
}

\author{
Joseph W Howells', Nikhil A Huprikar ${ }^{2}$, Dennis L Barnett II $^{1}$ \\ 1. Department of Medicine, Madigan Army Medical Center, Tacoma, WA, USA. 2. Department of Medicine, San Antonio \\ Uniformed Services Heath Education Consortium (SAUSHEC), San Antonio, TX, USA.
}

Correspondence: J oseph W Howells. Address: Department of Medicine, Madigan Army Medical Center, 7142 Axis St SE, Lacey, WA 98513, USA. E-mail: joseph.w.howells.mil@mail.mil

Received: August 7, 2015

DOI : $10.5430 /$ crim.v2n4p33

Accepted: September 14, 2015 Online Published: September 28, 2015

URL: http://dx.doi.org/10.5430/crim.v2n4p33

\section{Abstract}

A 72-year-old man with a past medical history significant for chronic obstructive pulmonary disease (COPD), witnessed nocturnal apnea and persistent 80-pack-year tobacco use presented with a new-onset of aphasia. MRI brain demonstrated restricted perfusion in the left temporoparietal area and insular cortex, consistent with subacute embolic strokes. Investigation for the etiology was notable for secondary polycythemia and non-specific mitral valve thickening on transthoracic echocardiography (TTE). Subsequent transesophageal echocardiography (TEE) showed a heterogenous, $1.1 \mathrm{~cm}$ mass on the anterior mitral valve leaflet. Following warfarin bridge therapy, surgical resection revealed a sterile thrombus, consistent with nonbacterial thrombotic endocarditis (NBTE). Chronic hypoxia has been implicated in the pathogenesis of NBTE in both human and animal studies. While animal models have suggested a direct causal relationship, human studies are largely limited to isolated case reports, which have almost uniformly been confounded by the presence of other disorders of hypercoaguability, such as malignancy or disseminated intravascular coagulation (DIC). Unique in comparison to prior studies, this report demonstrates a case of NBTE in the setting of chronic oxygen deprivation and secondary polycythemia in a patient without any additional major risk factors.

\section{Keywords}

Nonbacterial thrombotic endocarditis, Hypoxia, Polycythemia

\section{I ntroduction}

Nonbacterial endocarditis (NBTE) is characterized by the development of sterile valvular vegetations ${ }^{[1]}$. In addition to well-known risk factors such as advanced malignancy and antiphospholipid syndrome, there is evidence linking NBTE with hypoxic respiratory disease ${ }^{[2]}$. We present a case of NBTE associated with chronic oxygen deprivation secondary to chronic obstructive pulmonary disease (COPD), continued smoking, and probable obstructive sleep apnea.

\section{Case presentation}

A 72-year-old man presented to the emergency department with a 24-hour history of confusion and difficulty in speaking. His past medical history was notable for COPD (diagnosed four years prior with mild obstruction on pulmonary function 
studies and prescribed fluticasone/salmeterol with intermittent compliance), stage I colon cancer (resected six years prior with no evidence of recurrence on repeat colonoscopy), hypertension, hyperlipidemia and an active 80-pack-year tobacco smoking history. His only medications were omeprazole and aspirin, which he took intermittently.

On presentation, his vital signs were notable for blood pressure of 197/108, with normal pulse and temperature. His oxygen saturation was initially within normal limits on room air, however after falling asleep it quickly dropped to $89 \%$, requiring 2 liters of oxygen per minute via nasal cannula.

Physical examination revealed receptive aphasia, with otherwise intact strength, sensation, and cranial nerves. Cardiac auscultation revealed no murmurs, rubs or gallops, and extremities showed no clubbing or cyanosis. EKG revealed normal sinus rhythm, with no ST elevations or depressions.

On admission complete blood count revealed marked polycythemia, with a hemoglobin of $21.9 \mathrm{~g} / \mathrm{dl}$ and hematocrit of $65.8 \%$ (19.1 g/dl/56.2\% 17 months prior), with normal white blood cell count and platelets of $116 \times 10^{9} / \mathrm{L}$. Further workup of the erythrocytosis revealed negative JAK2 V617F mutation and normal erythropoietin (EPO) of 4.3 IU/L, consistent with secondary polycythemia. CT abdomen, chest, pelvis was negative for significant pathology.

MRI brain revealed focused areas of restricted diffusion in the left temporoparietal region and insular cortex (see Figure 1), which correlated with an M2 branch occlusion of the middle cerebral artery (see Figure 2), consistent with subacute cerebrovascular accidents (CVAs).
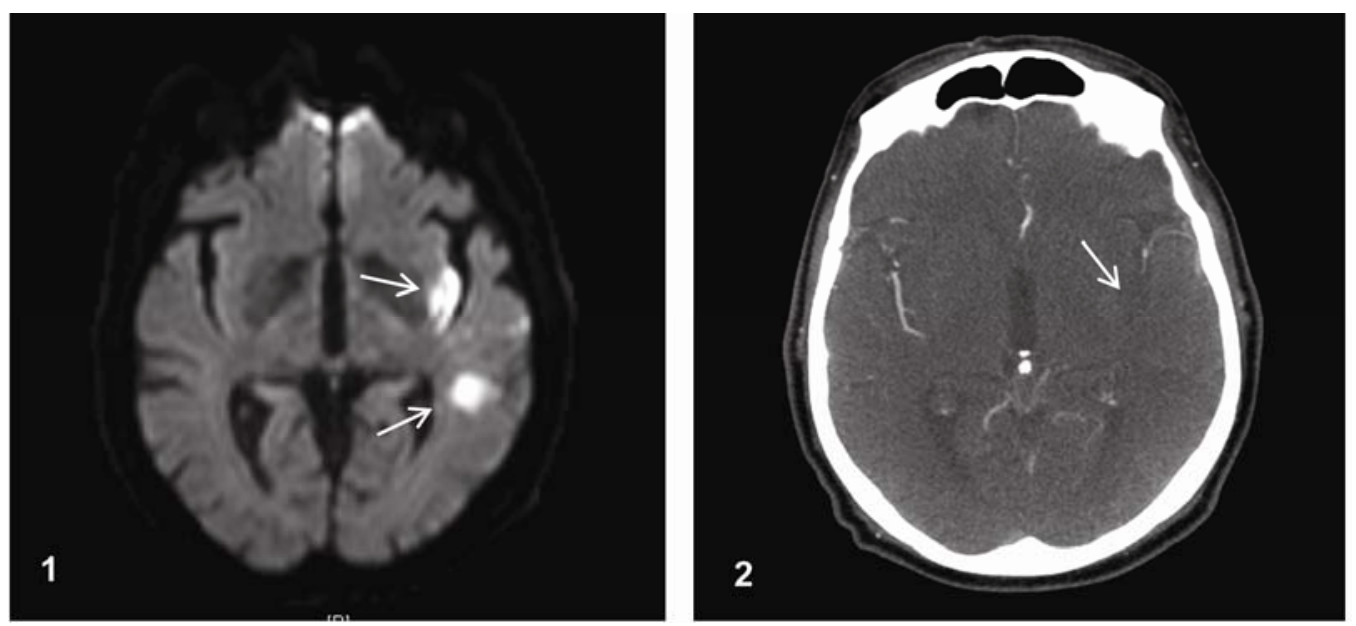

Figure 1. Diffusion weighted magnetic resonance imaging revealed marked areas of hyperintensity in the left temporoparietal region and insular cortex (arrows), consistent with ischemia. Figure 2. CT angiography revealed occlusion left M2 branch of the middle cerebral artery

Transthoracic echocardiography (TTE) revealed non-specific thickening of the mitral valve (see Figure 3). Subsequent transesophageal echocardiography (TEE) demonstrated a mobile, heterogeneously echoreflectant $1.1 \mathrm{~cm} \times 1.1 \mathrm{~cm}$ mass attached to mitral valve (see Figure 4).

Given the size of the lesion and lack of endocarditis risk factors or infectious symptoms, the initial concern was for a cardiac papillary fibroelastoma. After a preoperative cardiac catheterization, the patient was anticoagulated with warfarin and scheduled for surgical excision, which revealed an organized thrombus with no evidence of infection or valvular damage, consistent with NBTE. The patient tolerated the procedure well; however, post-operatively he developed atrial fibrillation, requiring long-term anticoagulation. He has had no recurrent thromboembolic events upon our last update. 


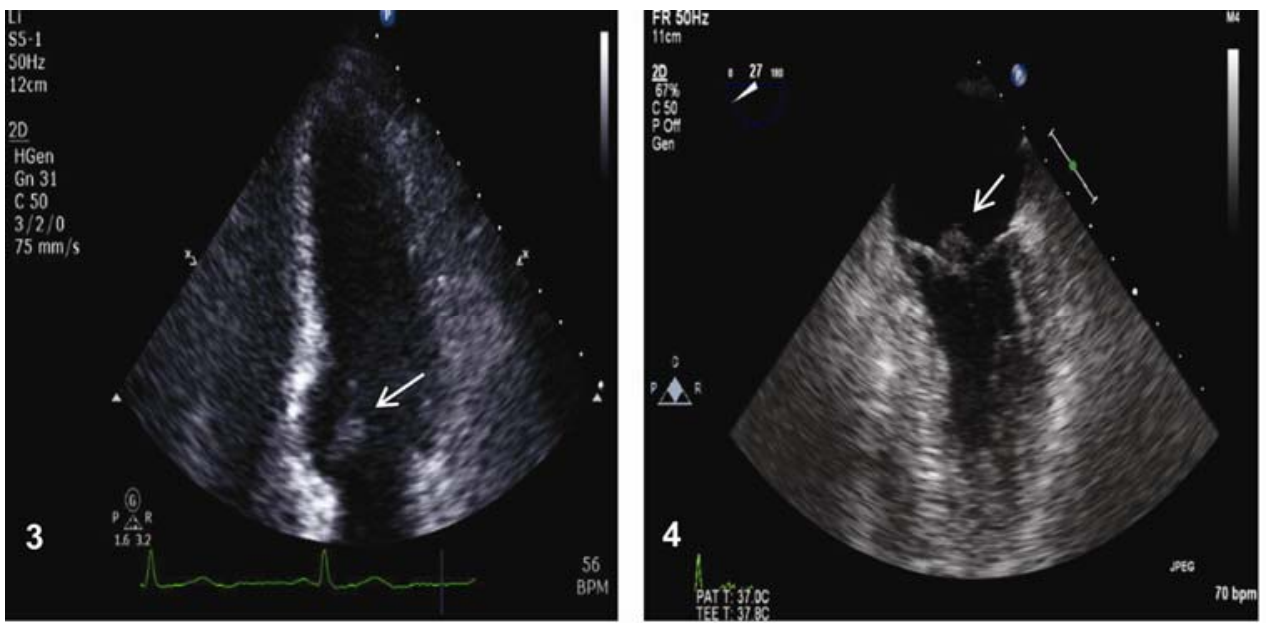

Figure 3. Transthoracic echocardiography (TTE) revealed nonspecific thickening of the mitral valve. Figure 4. Transesophageal echocardiography (TEE) revealed a $1.1 \mathrm{~cm} \times 1.1 \mathrm{~cm}$ heterogeneously echoreflectant mass adhering to the atrial side of the anterior and posterior leaflets of the mitral valve

\section{Discussion}

NBTE is characterized by the development of sterile thrombi on undamaged heart valves in the absence of infection ${ }^{[1]}$. Associated with hypercoaguable states, NBTE most commonly occurs in the setting of advanced malignancy and autoimmune disorders, such as antiphospholipid syndrome ${ }^{[2]}$. Given the superficial nature of the lesions, NBTE carries a high risk for systemic thromboembolic events, which can occur in up to $50 \%$ of patients ${ }^{[3]}$. On echocardiography, organized thrombi generally present as small, irregular vegetations, which are typically less than $1 \mathrm{~cm}$ in size ${ }^{[2]}$.

The differential diagnosis for mitral valve lesions on echocardiography includes both vegetations (infectious or nonbacterial) and primary cardiac tumors ${ }^{[3]}$. Bacterial endocarditis should always be excluded in this setting, given its' high prevalence and the potentially serious consequences of misdiagnosis. This patient had no infectious endocarditis risk factors (e.g., intravenous drug abuse, immunosuppression, history of endocarditis or prosthetic valves), along with normal white blood cell count, temperature, and 0/4 systemic inflammatory response syndrome (SIRS) criteria, making infectious endocarditis highly unlikely in this case. This was confirmed with surgical pathology, which revealed a bland thrombus with no evidence of lymphocytic infiltration, consistent with the diagnosis of NBTE.

In addition to autoimmune disease, advanced malignancy, and disorders of hypercoaguability, there is also evidence linking hypoxia to the pathogenesis of NBTE ${ }^{[2]}$. Some of the earliest studies showing this association came from animal models in the 1950s, where rats exposed to hypobaric chambers to simulate high altitudes developed sterile valvular vegetations ${ }^{[4]}$, suggesting a causative relationship between artificially-induced hypoxia and NBTE.

While the exact mechanism remains unknown, research suggests that calpain, a protease implicated in platelet reactivity, plays an important role in the pathogenesis of hypoxia-induced thrombogenesis ${ }^{[5]}$. Tyagi et al. found that rats exposed to simulated hypoxic environments had increased platelet reactivity associated with elevated calpain activity, which resolved when the protein was inhibited. It was also noted that patients with altitude-induced thrombosis had elevated calpain activity levels compared to matched controls ${ }^{[5]}$. Tissue factor (TF), a protein central to the initiation of the coagulation cascade, also likely plays a central role in the pathogenesis of NBTE. In animal models, rats with hypoxia-induced NBTE were noted to have significantly elevated tissue factor mRNA levels in foamy macrophages ${ }^{[6]}$. In human studies, patients with COPD exacerbations have been noted to have elevated tissue factor levels compared to healthy controls ${ }^{[7]}$.

It remains unclear whether secondary polycythemia contributes to the development of hypoxia-induced thrombosis. Though polycythemia vera is an established risk factor for NBTE ${ }^{[8]}$, the evidence linking secondary polycythemia with 
NBTE remains limited. In a case control study examining the prevalence of venous thromboembolisms (VTE) in COPD patients, Nadeem et al. found no significant differences in VTE rates between patients with secondary polycythemia and those with normal cell counts ${ }^{[9]}$. However, it should be noted that this was a relatively small study $(\mathrm{N}=86)$, with an average hematocrit of $53.5 \%$ in the polycythemia group (range $50.1 \%-63.4 \%$ ) ${ }^{[9]}$, compared to a hematocrit of nearly $66 \%$ in this case.

This patient, whose only other identified risk factors for NBTE aside from hypoxia were his age and remote history of localized colon cancer, represents an anomaly compared to previously published case reports, where the link between hypoxia and NBTE has almost always been confounded by the presence of other major risk factors, such as disseminated intravascular coagulation (DIC) and autoimmune disorders ${ }^{[10]}$. One possible explanation may be the magnitude of his polycythemia, suggesting a profound and chronic state of oxygen deprivation dating back at least 17 months prior. In animal studies, rats exposed to hypoxic environments had an incidence of NBTE of $33 \%$ at 4 weeks, compared to $100 \%$ at 12 weeks ${ }^{[6]}$, suggesting that the duration of hypoxia plays a pivotal role in the development of NBTE. It should also be noted that a full hypercoaguable workup was not performed in this patient, leaving the possibility of an underlying clotting disorder not entirely excluded.

Summarizing the evidence above, it appears that while a direct causal relationship has yet to be established, hypoxia, at a minimum, appears to play a facilitative role, augmenting the development of NBTE in patients with pre-existing risk factors, such as advanced malignancy or autoimmune disorders. Thus, as this case demonstrates, a high index of suspicion for NBTE should be maintained in patients with valvular masses in the setting of chronic oxygen deprivation. Further studies may seek to establish a definitive primary relationship between chronic hypoxia and the risk of developing NBTE, as well the role of hypoxia-induced polycythemia on NBTE pathogenesis.

\section{Acknowledgements}

Christina Schofield, MD (Madigan Army Medical Center, Tacoma, WA);

Eric Shry, MD (Madigan Army Medical Center, Tacoma, WA);

David Trowbridge, MD (Alaska Native Medical Center, Anchorage, AK).

\section{Disclaimer}

The views expressed are those of the authors and do not reflect the official policy of the Department of the Army, the Department of Defense or the U.S. Government.

\section{References}

[1] El-Shami K, Griffiths E, Streiff M. Nonbacterial thrombotic endocarditis in cancer patients: pathogenesis, diagnosis, and treatment. Oncologist. 2007; 12(5): 518-23. http://dx.doi.org/10.1634/theoncologist.12-5-518

[2] Asopa S, Patel A, Khan OA, et al. Non-bacterial thrombotic endocarditis. Eur J Cardiothorac Surg. 2007; 32(5): 696-701. http://dx.doi.org/10.1016/j.ejcts.2007.07.029

[3] Markides V, Nihoyannopoulos P. Non-bacterial thrombotic endocarditis. Eur J Echocardiography. 2000; 1(4): 291-4. http://dx.doi.org/10.1053/euje.2000.0027

[4] Highman B, Atland PD. Effect of altitude and cobalt polycythemia, hypoxia, and cortisone on susceptibility of rats to endocarditis. Circ Res. 1955; 3: 351-6. http://dx.doi.org/10.1161/01.RES.3.4.351

[5] Tyagi T, Ahmad S, Gupta N, et al. Altered expression of platelet proteins and calpain activity mediate hypoxia-induced prothrombotic phenotype. Blood. 2014; 123(8): 1250-60. http://dx.doi.org/10.1182/blood-2013-05-501924

[6] Nakanishi K, Tajima F, Nakata Y, et al. Tissue factor is associated with The nonbacterial thrombotic endocarditis induced by a hypobaric hypoxic environment in rats. Virchows Arch. 1998; 433(4): 375-9. http://dx.doi.org/10.1007/s004280050262

[7] Wang Y, Zheng Y, Zhai YL, et al. Comparative analysis of MCP-1 and TF in elderly patients with acute exacerbations of COPD and its clinical significance. Eur Rev Med Pharmacol Sci. 2015; 19(2): 215-19. PMid:25683933 
[8] Reisner SA, Rinkevich D, Markiewicz W, et al. Cardiac involvement in patients with myeloproliferative disorders. Am J Med. 1992; 93(5): 498-504. http://dx.doi.org/10.1016/0002-9343(92)90576-W

[9] Nadeem O, Gui J, Ornstein DL. Prevalence of venous thromboembolism in patients with secondary polycythemia. Clin Appl Throm Hemost [Internet]. 2013 [cited 2015 Jul 17]; 19(4). http://dx.doi.org/10.1177/1076029612460425

[10] Young RS, Zalneraitis EL. Marantic endocarditis in children and young adults: clinical and pathological findings. Stroke. 1981; 12(5): 635-9. http://dx.doi.org/10.1161/01.STR.12.5.635 